\title{
MATHEMATICAL MODELLING OF PYROLYSIS OF HARDWOOD (ACACIA)
}

\author{
Alok DHAUNDIYAL ${ }^{1 *}$, Suraj B. SINGH ${ }^{2}$, Istvan BACSKAI ${ }^{3}$ \\ 'Institute of Process Engineering, Szent István University, Godollo, Hungary \\ ${ }^{2}$ Govind Ballabh Pant University of Agriculture and Technology, Pantnagar, Uttarakhand, India \\ ${ }^{3}$ Institute of Agricultural Mechanisation, National Agriculture and Innovation Centre, Godollo, Hungary
}

\begin{abstract}
This paper emphasises the analogous modelling of hardwood (acacia) pyrolysis. The impacts of physical characteristics of hardwood chips on the pyrolysis are examined through the conservation of biomass solid mass fraction. The ONORM standard chips of sizes ' $G 30$ ' and ' $G 50$ ' and their combination are individually tested in the pyrolysis reactor. In the analogous situation, the fixed bed is assumed to be a wooden slab with a porosity equivalent to the voidage of bed. Bulk density, bed length and porosity are several of the physical attributes of a fixed bed used to examine the variation in the hardwood solid mass across the fixed bed. To measure temperature, the four-temperature sensors separated from each other by $80 \mathrm{~mm}$ are fixed along periphery of a reactor. The heating element of $2 \mathrm{kWe}$ is provided to initiate the biomass pyrolysis. The proposed model is also used to establish the relationship between the kinetics of pyrolysis and the structural properties of hardwood.
\end{abstract}

Keywords: pyrolysis; heat transfer; wood chips; physical structure; kinetics

The pyrolysis process plays an important role in comprehending the intrinsic mechanism of combustion of wood and sawdust, since the released products - char and volatile matter - further undergo smouldering (glowing combustion) and flaming (light-emitting) combustion, respectively, to release thermal energy. The quality of char production and generation of volatile matter depends on the physical state of biomass before undergoing thermal decomposition. Therefore, it becomes indispensable to focus on the relationship between the structural properties of biomass and thermal behaviour of biomass. The main aim of any model is to provide a computational inference for examining the system parameters and to recognise the relevance of system characteristics for knowing the system behaviour. Although the complexity of the thermochemical process makes the computational process cumbersome, it can be resolved using some cemented steps while formulating the proposed model. The pyrolytic decomposition of wood comprises the series of reactions, therefore, the altering thermal condition or characteristics of fuel may not only influence the rate of reaction but also the pathway of reactions.

The different perspective of modelling is proposed through the application of conservation of energy associated with solid biomass. The relationship between physical and thermal characteristics of wood and their influence on the decomposition process has been highlighted by the analogous scheme. However, the various modelling scheme pivoting around the kinetic aspect of the pyrolysis are summarised in the literature (Dhaundiyal et al., 2018a; Dhaundiyal et al., 2018b; Dhaundiyal et al. 2018c; Cai and Lui, 2008; Dhaundiyal et al., 2019a; Mani et al., 2009). It is assumed that the fixed bed behaves like a porous wooden slab, the length of which sinks with time as a shrinking radius of the particle during combustion (Fogler, 2004). The objective of this study is to evaluate concurrently the thermogravimetric behaviour of the wooden chips with varying physical properties of the system that changes with time and temperature and develop an analogous model under the given thermal conditions for a fixed bed.

\section{Material and methods}

\section{Modelling scheme}

Material characteristics have vital importance during the conceptual stage of modelling. Generally, the thermal parameters (diffusivity and thermal conductivity) directly influence the process of pyrolysis. The physical factors (bulk density and temperature of material) can easily be related to heat and mass transfer properties by the basic experiments. On the other hand, heat conduction through the porous medium varies with the direction of heat flow across the biomass. Therefore, the overall thermal conductivity is the summation of thermal conductivities of the air-void spaces and the solid biomass. Aforementioned process can be followed to measure the heat capacity and the heat transfer coefficient. Wood anisotropy can be known through the fact that permeability of fluid along the grains is $10^{4}$ times higher than across the grain, whereas thermal conductivity along the grain is twice over the thermal conductivity across the grain (Roberts and Clough, 1963). It is reported that the thermal conductivity of pyrolysed material is a linear function of thermal conductivity of the

Contact address: Alok Dhaundiyal, Institute of Process Engineering, Szent István University, Godollo, Hungary; e-mail: Alok.dext@hotmail.com 
virgin wood and char, which is related to an instantaneous density of wood. It is noticed that the temperature and mass loss predictions are sensitive to the assumed value for the thermal conductivity of char (Kung, 1972). However, the measurement of thermal parameters at very high temperature is quite complicated due to chemical alteration and the drastic variation in the physical properties makes computational work much complex. Certain heat and mass transfer models, which combine the chemical kinetics with the application of heat transfer, are proposed to determine the effect of thermal parameters on the thermo-kinetics of material (Maa and Bailie, 1973). One of such models, a volume reaction model, emphasises the simultaneous heat and mass transfer in the particle. In the unreacted shrinking core model for high temperatures (Maa and Bailie, 1973), the reaction at an unreacted shrinking core of solid is initiated by the surrounded pyrolysed layer of material around it. The reaction takes place at the interface of these two solid regions; therefore, the conduction mode plays a major role in dissipation of the thermal energy from the charred layer. The energy transferred during this autocatalytic process varies with the temperature gradient and the radius of the unreacted core. The same energy is utilised to commence pyrolysis in the biomass. The gases produced during pyrolysis advance radially outward. The unsteady rise of temperature inside particles is a time function of porosity, which helps to distinguish the variation of internal energy change of the gas and solid. A gas-solid thermal equilibrium can only be maintained for the low velocity of fluid flow across material (Dhaundiyal et al., 2020). Another aspect of heat transfer is to determine the thermal profile required as an explanatory variable in the kinetic model. Thus, the information on the heat transfer parameters assists in evaluating the accuracy of the prediction of the overall model. Several other models have also incorporated the internal convective heat transfer and temperature dependent thermal conductivity (Witkowski et al., 2016; Sadhukhan et al., 2009; Polesek and Kardas, 2015). In this study, a wooden slab, which is analogous to a fixed bed, is subjected to transfer heat across slab along the length $L$. The schematic diagram of the assumed wooden slab is given in Fig. 1. The length of bed is $80 \mathrm{~mm}$, so the equivalent length of bed with respect

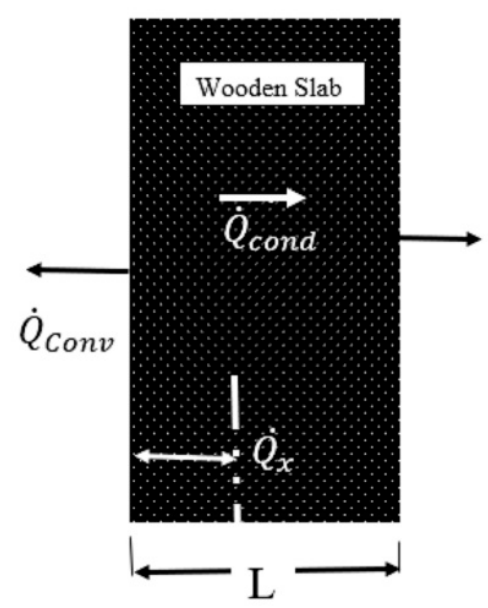

Fig. 1 Schematic diagram of heat transfer across the wooden slab to $G 30$ and $G 50$ are derived by the algorithm. In actual situation, porosity increases with time and temperature. But for the sake of model simplicity, the porosity has been kept constant. For comparison, parameters are assumed to be fixed in modelling problem, so that relative variation can be measured.

According to the conservation of the solid energy (Eq. $1)$, the algebraic sum of the change of internal energy of solid (I), the heat conduction through the fixed bed (II), the internal convective heat transfer between fixed bed and the ambience (III), and the heat of reaction within the solid (IV) is equal to zero:

$$
\underbrace{\Delta U}_{I}+\underbrace{\dot{Q}_{\text {conduction }}}_{I I}+\underbrace{\dot{Q}_{\text {convection }}}_{I I I}-\underbrace{\Delta H_{\text {heatofreaction }}}_{I V}=0
$$

Simplification of Eq. (1) in time basis can be expressed as:

$$
\begin{aligned}
& \frac{\partial\left\{h_{s} \rho_{s}(t)(1-\varepsilon)\right\}}{\partial t}-\left(k_{s}(1-\varepsilon) \frac{\partial T}{\partial x}\right)_{x=L}+ \\
& +\frac{\bar{h}}{(1-\varepsilon)}\left(\theta_{0}\right)-\frac{\partial\left\{C_{p} \rho_{s}(t)(1-\varepsilon) T\right\}}{\partial t}=0
\end{aligned}
$$

where:

$\theta_{0}=\left(T_{s}-T_{g}\right)$

$$
G=\sqrt{\frac{h_{s} P}{k_{s} A}}
$$

$$
\begin{gathered}
\frac{h_{s}}{k_{s}}\left(\frac{\partial\left\{\rho_{s}(t)(1-\varepsilon)\right\}}{\partial t}\right)-(1-\varepsilon)\left(G \frac{\sin h\left[G\left(x-L_{c}\right)\right]}{\cos h\left[G\left(L-L_{c}\right)\right]}+\frac{\partial T_{g}}{\partial x}\right)+ \\
+\frac{\overline{N u}}{L} \frac{1}{(1-\varepsilon)}\left(\frac{\cos h\left[G\left(x-L_{c}\right)\right]}{\cos h\left[G\left(L-L_{c}\right)\right]}\right)-\frac{1}{\alpha_{s}} \frac{\partial\{T\}}{\partial t}=0
\end{gathered}
$$

By integration of Eq. (3) with respect to time (t), one gets expression for mass losses during pyrolysis for the combination of $G 30$ and $G 50$ chips:

$$
\begin{gathered}
m_{c}(t)=0.001 m_{c}\left(t_{0}\right)-\left[0 . 0 0 0 8 6 4 \left[L^{2}\left(-0.0045 \cdot L^{2}+0.267 \cdot L\right)-\right.\right. \\
\left.-L_{c}^{2}\left(-0.0018 L \cdot L_{c}+0.8013 L+0.00135 L_{c}^{2}-0.534 L_{c}\right)\right]+ \\
+0.000864\left[G \left\{\left(L \cdot L_{c}-2-L_{c}^{2}\right) \operatorname{sech}\left(G\left(L-L_{c}\right)\right)-\right.\right. \\
\left.-1,000 L \cdot \tanh \left(G\left(L-I_{c}\right)\right)\right\}+0.28\left\{L+\left(L-2 L_{c}\right) \cdot \operatorname{sech}\left(G\left(L-L_{c}\right)\right)-\right. \\
\left.\left.\left.-2 \tanh \left(G\left(L-L_{c}\right)\right)\right\}\right]\right]
\end{gathered}
$$

Similarly, the deduced expressions for mass losses in pyrolysis of hardwood chips $G 30$ and G50 are given by Eq. (5) and Eq. (6), respectively:

$$
\begin{gathered}
m_{G 30}(t)=0.001 m_{G 30}\left(t_{0}\right)-\left[0 . 0 3 2 \left[L^{2}\left(0.3847 L-0.00068 L^{2}\right)-\right.\right. \\
\left.+L_{c}^{2}\left(0.00205 L_{c}^{2}+1.154 L-0.00273 L L_{c}-0.7694 L_{c}\right)\right]+ \\
+0.032\left[G \left\{\left(L \cdot L_{c}-2-L_{c}^{2}\right) \operatorname{sech}\left(G\left(L-L_{c}\right)\right)-1,000 L\right.\right. \\
\left.\cdot \tanh \left(G\left(L-I_{c}\right)\right)\right\}+0.28\left\{L+\left(L-2 L_{C}\right) \cdot \operatorname{sech}\left(G\left(L-L_{c}\right)\right)-\right. \\
\left.\left.\quad-2 \cdot \tanh \left(G\left(L-L_{c}\right)\right)\right\}\right] \\
m_{G 50}(t)=0.001 m_{G 50}\left(t_{0}\right)-\left[0 . 0 0 8 8 9 \left[L^{2}\left(0.2208 L-0.00033 L^{2}\right)-\right.\right. \\
\left.-L_{c}^{2}\left(0.001 L_{c}^{2}-0.441 L_{c}+0.662 L-0.00133 L L_{c}\right)\right]+ \\
+0.00889\left[G \left\{\left(L \cdot L_{c}-2-L_{c}^{2}\right) \operatorname{sech}\left(G\left(L-L_{c}\right)\right)-1,000 L \cdot\right.\right. \\
\left.\cdot \tanh \left(G\left(L-I_{c}\right)\right)\right\}+0.28\left\{L+\left(L-2 L_{c}\right) \cdot \operatorname{sech}\left(G\left(L-L_{c}\right)\right)-\right. \\
\left.\left.\quad-2 \cdot \tanh \left(G\left(L-L_{c}\right)\right)\right\}\right]
\end{gathered}
$$


where:

$L \quad$ - the equivalent length of the fixed bed (m)

$L_{c} \quad$ - the corresponding length of the shrinking bed $(\mathrm{m})$

$G$ - geometric parameter $\left(\mathrm{m}^{-1}\right)$

$B$ - Biot number

$\rho_{s} \quad-$ density of solid mass $\left(\mathrm{kg} \cdot \mathrm{m}^{-3}\right)$

$T_{g} \quad$ - temperature of the volatile gas $\left({ }^{\circ} \mathrm{C}\right)$

$T_{s} \quad$ - temperature of the solid mass $\left({ }^{\circ} \mathrm{C}\right)$

$\varepsilon \quad$ - voidage of bed

$h \quad$ - heat transfer coefficient $\left(\mathrm{kW} \cdot \mathrm{m}^{-2} \cdot \mathrm{K}^{-1}\right)$

$k_{s} \quad-$ thermal conductivity $\left(\mathrm{kW} \cdot \mathrm{m}^{-1} \cdot \mathrm{K}^{-1}\right)$

$\mathrm{Nu}$ - Nusselt number

$\theta_{0}$ - temperature difference between solid and the volatile gas $\left({ }^{\circ} \mathrm{C}\right)$

$\alpha_{s} \quad-$ thermal diffusivity of solid $\left(\mathrm{m}^{2} \cdot \mathrm{s}^{-1}\right)$

$m$ - mass $(\mathrm{g})$

$P$ - perimeter of the cross sectional area of a slab (m)

A - cross sectional area of a slab $\left(\mathrm{m}^{-2}\right)$

$h_{s} \quad$ - internal energy of the solid mass $\left(\mathrm{kJ}^{\mathrm{kgg}}{ }^{-1}\right)$

The relationship between the rate of change of bulk density activation energy of wood is given by Eq. (7):

$$
\frac{d\left(\frac{\rho_{b(t)}}{\rho_{b\left(t_{0}\right)}}\right)}{d t}=-A \cdot \exp (-E / R T)\left|\rho_{b(t)}-\rho_{b\left(t_{0}\right)}\right|
$$

where:

$$
\begin{aligned}
A_{i} & - \text { frequency factor } \\
E \quad- & \text { activation energy necessary for thermal } \\
& \text { decomposition of hardwood }
\end{aligned}
$$

\section{Experimental set-up}

The experimental testing rig is situated at the National Agriculture Research and Innovation Centre, Hungary. The different sizes of the hardwood chips (G30 and G50) (Austrian Standard International (ÖNORM), Austria) are used for the experimental purposes. The physiochemical properties of hardwood are determined using physical and chemical analysers. To measure the system extensive and intensive properties, the thermogravimetric, pressure, and temperature sensors are retrofitted around the periphery of the pyrolysis reactor. The illustrative diagram of reaction is shown in Fig. 2 (Nickel-Chromium/Nickel-Alumel). The weight sensor, which is separated by the insulating cover, is kept under the heating chamber. All the sensors are connected to the computer through a data logger (HBM, Germany). The volumetric rate of inert gas is controlled by a flow meter. The indirect heating element (Herz-filament, Hungary) of $2 \mathrm{kWe}$ is installed inside the reactor to initiate pyrolysis process of biomass. The grid material of heating chamber is made of $0.7 \mathrm{~mm}$ stainless steel, while the rock wool of $50 \mathrm{~mm}$ thickness is coated around the reactor. The

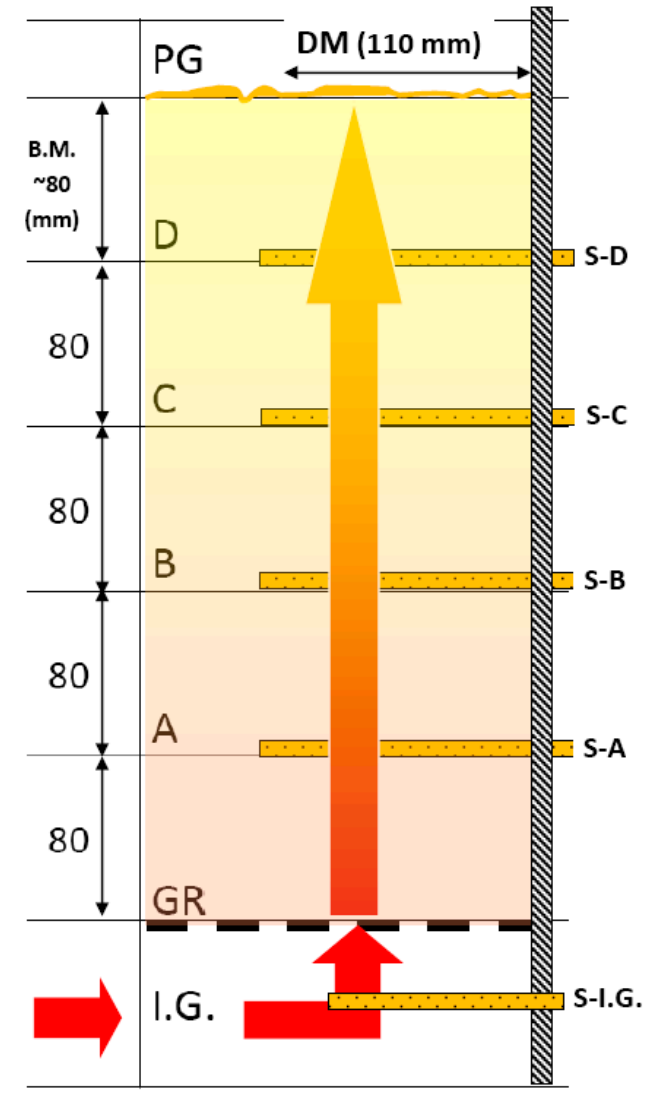

Fig. 2 Schematic diagram of pyrolysis reactor (S-A, S-B, S-C, $S-D$, temperature sensors; $S-I-G$, thermogravimetric sensor; GR, grate material)

core of the pyrolysis chamber is made of $1.5 \mathrm{~mm}$ welded carbon steel. The inner diameter of reactor is $110 \mathrm{~mm}$, the outer diameter is $210 \mathrm{~mm}$. The material used to fabricate the reactor periphery is aluminium. Elemental composition of the raw material is determined by CHN-S analyser (VARIO $E L$, Italy). The analyser gets heated up to temperature of $2000{ }^{\circ} \mathrm{C}$ before the process initialisation. The helium gas is used as a carrier gas inside CHN-S analyser; its function is to carry away the product of combustion to different reduction columns. These tubes are placed in-between the combustion chamber and the signal-processing unit. The gases are separated into their constituents through purge or trap chromatography. After this process, each component is individually detected by a thermal conductivity detector (TCD, Thermo Fisher, USA). The product of combustion is absorbed in sequence; however, nitrogen does pass through the reduction columns. The physiochemical characteristic of hardwood (acacia) is illustrated in Table 1 (dry basis). The kinetic parameters are determined using Eq. (7). The area under the predicted curves is evaluated using the MATLAB

\begin{tabular}{|c|c|c|c|c|c|c|c|c|}
\hline C \% & H \% & $\mathbf{N} \%$ & $\mathbf{S} \%$ & $0 \%$ & Ash \% & $\mathrm{HHV}^{*}\left(\mathrm{MJ} \cdot \mathrm{kg}^{-1}\right)$ & $\rho_{b}\left(\mathbf{k g} \cdot \mathrm{m}^{-3}\right)$ & $\rho\left(\mathbf{k g} \cdot \mathbf{m}^{-3}\right)$ \\
\hline 45.84 & 5.62 & 0.18 & 0.06 & 39.91 & 0.58 & 19.02 & 545 & 760 \\
\hline
\end{tabular}
(2015a) software.

Table 1 Physiochemical characteristic of hardwood (dry basis)

\footnotetext{
* higher heating value; $b$ - bulk density
} 


\section{Results and discussion}

An analogous model for hardwood pyrolysis is used to determine the effect of physical properties on decomposition of hardwood chips. Variation of the proposed geometrical parameter $G$ on the mass loss curves is illustrated in Fig. 3. The geometrical parameter $G$ is a dependant function of the thermal and geometric parameters of a wooden slab. However, the thermal properties are fixed for a particular material, therefore, it does not vary with a given condition. The slab dimensions are subjected to alterations so that the effect of geometry of a pyrolysis bed on the mass loss variation can be examined. It is known through the proposed modelling scheme that the increasing value of $G$ shifts the mass-loss curve upwards. It implies that the symmetrical slab allows more char production than that of the asymmetrical one. Moreover, the fractional reduction of the residual mass is relatively high due to asymmetricity. This is due to reason that a symmetrical slab lets the volatiles escape more quickly than the asymmetrical one, the thermal energy accompanying the released gas thus reduces the residence time of volatiles inside the matrix. Finally, it prevents autocatalytic reaction from occurring inside the matrix, hence the large size chip helps to develop the large thermal gradient, and it causes local condensation of volatiles. The similar attribute has also been reported for pyrolysis of a large particle (Dhaundiyal et al., 2020; Bartlett et al., 2019). The thermal gradient is also affected by the slab geometry. The thermal gradient across the wooden slab of

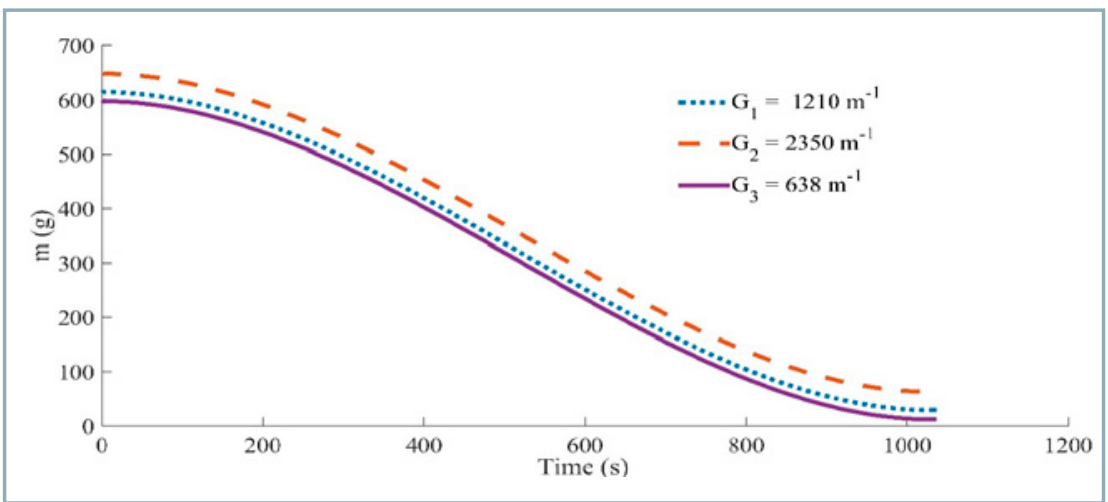

Fig. 3 Effect of the geometrical parameter ' $G$ ' on the numerical solution of pyrolysis of hardwood chips

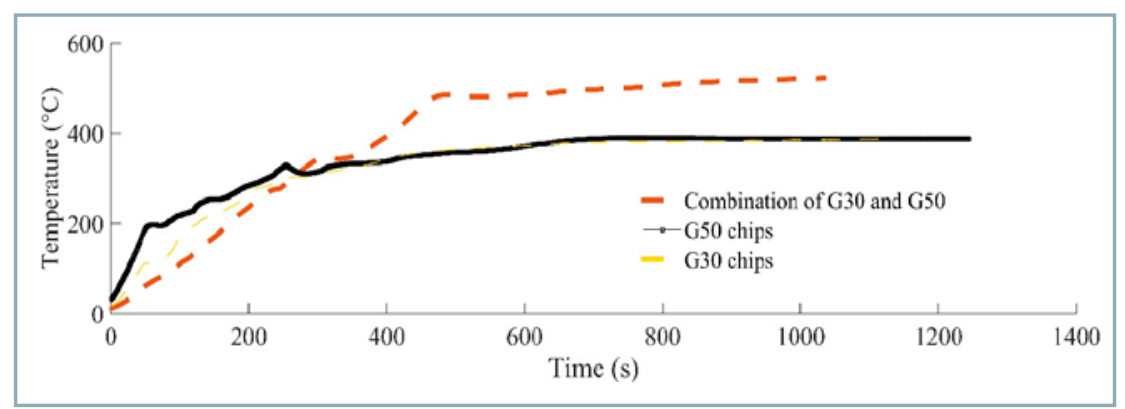

Fig. 4 Temperature distribution across pyrolysis bed of hardwood

Table 2 Properties of an equivalent wooden slab for the proposed model

\begin{tabular}{|l||c|c|c|c|}
\hline Hardwood Chips & $\boldsymbol{R}^{\mathbf{2}}$ & $\boldsymbol{L}(\mathbf{m})$ & $\boldsymbol{G}\left(\mathbf{m}^{-\mathbf{1}}\right)$ & $\boldsymbol{B}_{\boldsymbol{i}}$ \\
\hline \hline G30 & 0.99 & $0.0732 \leq L \leq 0.074$ & $G \leq 3590$ & 7.49 \\
\hline G50 & 0.97 & $0.095 \leq L \leq 0.108$ & $G \leq 7161$ & 10.32 \\
\hline Combination (G30 and G50) & 0.98 & $0.085 \leq L \leq 0.0889$ & $G \leq 5348$ & 8.84 \\
\hline
\end{tabular}

the $G 50$ chip is higher than that of the wooden slab of $G 30$ chip. The value of the Biot number $(B)$ varies from 7.49 to 10.32 for different size of hardwood chips (Table 2), which implies that temperature gradient across the slab is non-uniform. It is also established from the experimental results that the nonuniform distribution of temperature across the fixed bed does exist and it affects the mass variation of hardwood chips with respect to time (Fig. 4). Moreover, to determine whether the proposed scheme can be extrapolated, it is necessary to examine the higher thermal regime so that the application of the model can be demarcated.

The effect of equivalent length of slab is illustrated in Fig. 5. The slab length $L$, analogous to the height of the fixed bed of G30, G50, and combination of $G 30$ and G50, affects the decomposition of the wooden slab at the beginning of pyrolysis. The increasing length of the wooden slab causes the mass-loss scale to shift upward, which in turn decreases the rate of conversion of wood chips throughout the whole pyrolysis process. The relative change in the residual mass of wood due to variation of the equivalent length is lesser than the relative change in the initial mass of wood. It implies that the shrinkage of the wooden slab has a significant contribution to increase the convective heat transfer resistance across the wooden slab, thus the conversion rate is nearly the same for the varying size of wooden slab. It is noted that the same characteristics have been found for G30, G50 and the combination of both.

The effect of porosity on numerical solution is depicted in Fig. 6. The porosity is proportional to the volume fraction of the air void and solid mass. It is evident from the predicted solution that the increasing fraction of porosity of air-void makes the mass-loss curve shifted downward at the beginning of pyrolysis. The conversion rate tends to be more uniform as the porosity increases, therefore, it can be inferred that the fluid resistance to the volatile content increases. The resistance is relatively high in the large wooden slab; thus, the size of wooden slab may influence the extent of the secondary pyrolysis reactions, as well as the yield of char through mass transfer. The 

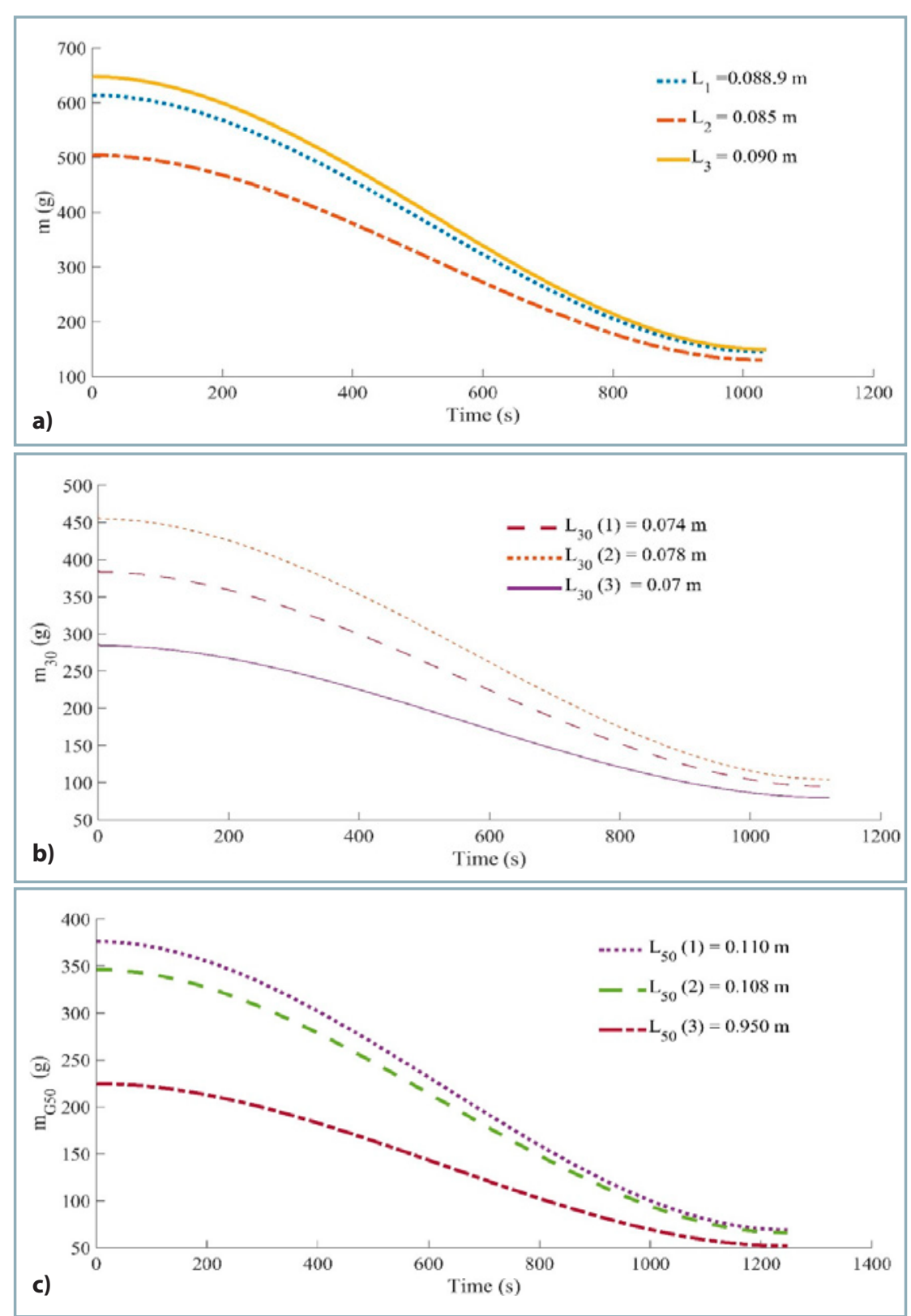

Fig. 5 Behaviour of mass loss curve with respect to the equivalent length of slab $L$ a - heterogeneous mixture of $G 30$ and G50; b - G30; c - G50 chips

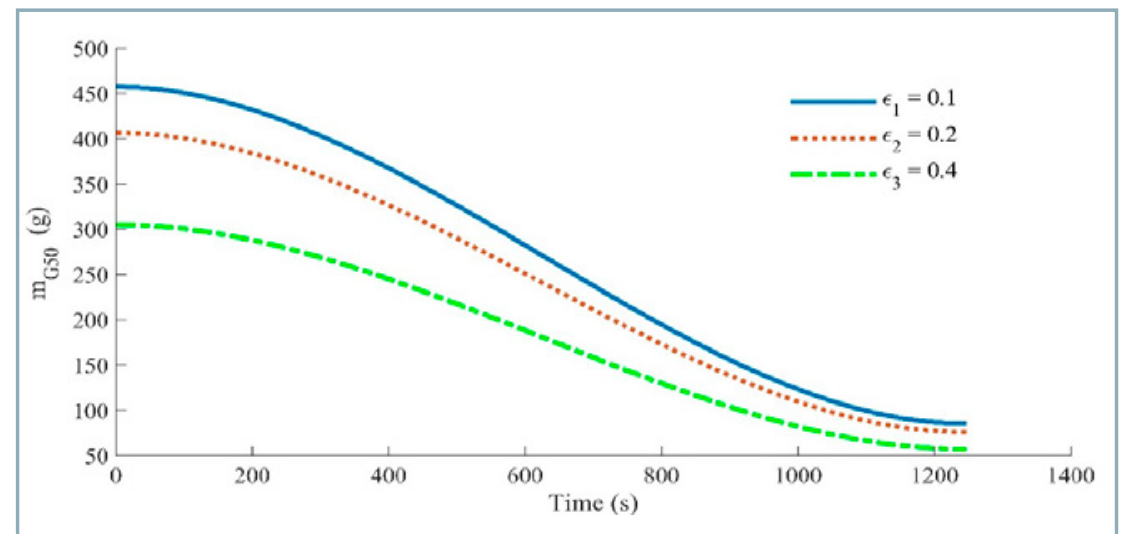

Fig. 6 Effect of porosity $(\varepsilon)$ on the numerical solution of hardwood pyrolysis direction of heat flow perpendicular to the grain orientation also increases the residence time of pyrolysis gases and supports the autocatalytic reactions (Roberts and Clough, 1963). The experimental plot of mass-loss of hardwood chips is illustrated in Fig. 7. The similar characteristic has been observed in the proposed model. The decomposition curve of hardwood chips, G50, has relatively linear attribute at the onset of pyrolysis that shows that thermal gradient across the fixed bed is larger than that of the fixed bed of G30 chips. Therefore, for the similar temperature interval, the domain of devolatisation is relatively extended for the large particle.

Comparison of the predicted solution for different wooden slab sizes with the experimental data is demonstrated in Fig. 8. It is clearly visible that the predicted solution provided the good correlation with the experimental curve. The assumption of the fixed bed as a wooden slab with the conversation of mass in hardwood pyrolysis depicts that the residual mass, range of devolatisation, convective resistance, residence time of volatiles in the matrix, and effect on conversion rate are highly influenced by physical fuel characteristics. The predicted solution shows good compliance at the initial stage of pyrolysis and the difference between residual masses of predicted and experimental is marginally low. On the other hand, the proposed models for $G 30$ and $G 50$ chips are depicted in Fig. 8 (b, c). Here, the numerical solution for $G 50$ has shown a slight deviation in the residual mass, whereas the deviation of the residual mass for $G 30$ is relatively low. This may be due to either non-uniformity of temperature gradient or the structural effects (surface cracks or longitudinal channelling), which occur due to the sudden drop of pressure inside the slab that leads to alteration of the heating characteristic of biomass.

The kinetic constants evaluated for different chip sizes are shown in Table 3. The estimated value of activation energy is compared with the different species of wood and it is within permissible range of variation (Lim and Chew, 2005). The activation energy for hardwood (acacia) varies from 73.5 to $88.48 \mathrm{~kJ} \cdot \mathrm{mol}^{-1}$, whereas the frequency factor varies from 

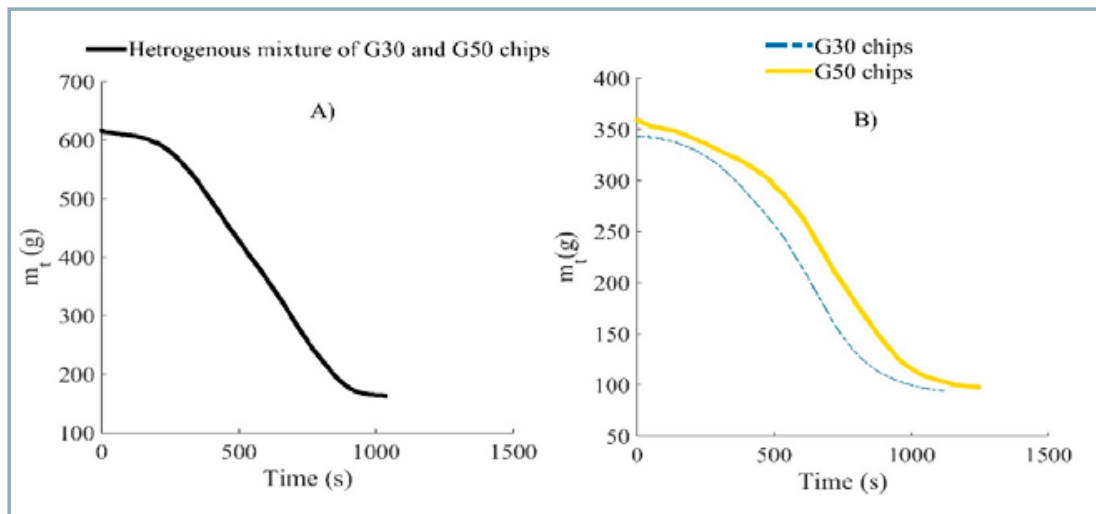

Fig. 7 Mass loss curves for the wooden chips

A - Heterogeneous mixture of $G 30$ and $G 50 ; B$ - G30 and G50 chips
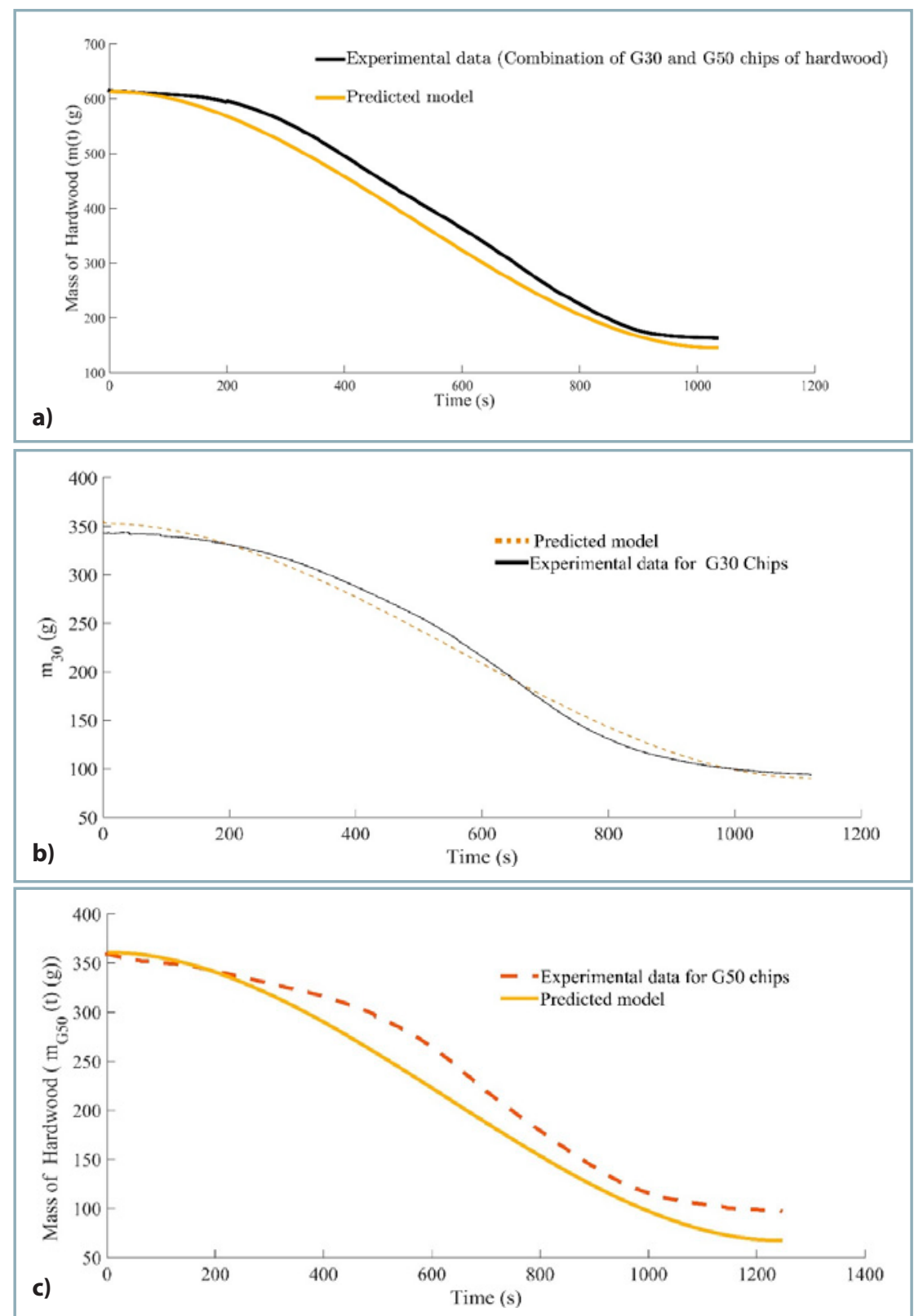

Fig. 8 Comparison of experimental data with the predicted solution for different combination of hardwood chips

a - heterogeneous mixture of G30 and G50; b - G30; c - G50 chips
$1.02 \times 10^{4}$ to $1.27 \times 10^{4} \mathrm{~min}^{-1}$. The detailed information is given in Table 3. It is found that the size variation of wooden slab also affects the kinetic study of pyrolysis, however, the range of temperature used for experimental purpose has a major role in determining the kinetic parameters. The difference between the kinetic parameters of wooden slabs of $G 30$ and $G 50$ chips is marginally narrow. The bulk density of bed and the porosity play a significant role in determining the apparent activation energy. It implies that the kinetic constants, as well as the heat of reaction, before and after the breaking point of wood, differ from each other (Tinney, 2007). It is noted that the Monte Carlo is implemented to measure the kinetic parameters (Dhaundiyal et al., 2019b; Dhaundiyal and Toth, 2020).

\section{Conclusion}

Pyrolysis modelling of hardwood (acacia) pivoted around the analogous model, which is equivalent to the fixed bed of pyrolysis reactor. The effect of physical properties on thermogravimetry of the wooden chips is investigated through a proposed model. It is found that the equivalent length of wooden slab for $G 30$ should vary from 73 to $74 \mathrm{~mm}$, whereas it is relatively large for $G 50$ and the combination of both of them. The wooden slab of $95-108 \mathrm{~mm}$ is suitable to simulate the result for $G 50$ chips. On the other hand, it is $85-89.5 \mathrm{~mm}$ for combination of both. The results simulated by proposed model provide a good correlation with experimental results (Table 2). Moreover, the kinetic constants for hardwood is found to vary from 73.5 to $88.48 \mathrm{~kJ} \cdot \mathrm{mol}^{-1}$, which also lie within a permissible range of variation of activation energy for different species of wood (MüllerHagedorn et al., 2003). The Biot number for the wooden slab varies from 7.49 to 10.49 , which implies that the temperature gradient is non-uniform across the wooden slab. Moreover, with respect to time, the variation of bulk density of bed also influences the thermo-kinetics of hardwood. It is concluded that kinetic constants, before and after the wood breakage point, may differ from each other. The limiting factor of this analysis focuses on the resistance to the flow of fluid and 
Table 3 Kinetic constants for hardwood (acacia)

\begin{tabular}{|l||c|c|c|c|}
\hline Area under curves & Chip Size & $\boldsymbol{E}$ & $\boldsymbol{A}_{\boldsymbol{i}}$ & Activation energy (kJ.mol $\left.^{-1}\right)^{*}$ \\
\hline $\mathbf{1 4 8 3 3 0}$ sq. unit & $G 30$ & $73.5 \mathrm{~kJ} \cdot \mathrm{mol}^{-1}$ & $1.20 \times 10^{4}\left(\mathrm{~min}^{-1}\right)$ & $71 \mathrm{~kJ} \cdot \mathrm{mol}^{-1}$ (beech saw dust) \\
\hline $\mathbf{1 9 0 9 4 0}$ sq. unit & $G 50$ & $74 \mathrm{~kJ} \cdot \mathrm{mol}^{-1}$ & $1.06 \times 10^{4}\left(\mathrm{~min}^{-1}\right)$ & $125.4 \mathrm{~kJ} \cdot \mathrm{mol}^{-1}$ (softwood) \\
\hline $\mathbf{2 5 6 6 1 0}$ sq. unit & combination & $88.48 \mathrm{~kJ} \cdot \mathrm{mol}^{-1}$ & $1.27 \times 10^{4}\left(\mathrm{~min}^{-1}\right)$ & $85.69 \mathrm{~kJ} \cdot \mathrm{mol}{ }^{-1}$ (hardwood), 55 (pine) \\
\hline
\end{tabular}

Source*: Lim and Chew, 2005; Blasi, 1993; Dhaundiyal and Singh 2019

the surface of the wooden slab. The convection resistance must be low and conduction resistance should be high, and it is clearly depicted by the Biot number. The decrease in the convection resistance must be higher than the increase in the conduction resistance for better correlation between the experimental and the mathematical model.

\section{References}

BARTLETT, A. I. - HADDEN, R. M. - BISBY, L. A. 2019. A review of factors affecting the burning behaviour of wood for application to tall timber construction. In Fire Technology, vol. 55, no. 1, pp. 1-49.

BLASI, C. D. 1993. Modeling and simulation of combustion processes of charring and non-charring solid fuels. In Progress in Energy and Combustion Science, pp. 71-104.

CAI, J. - LIU, R. 2008. New distributed activation energy model: Numerical solution and application to pyrolysis kinetics of some types of biomass. In Bioresource Technology, vol. 99, no. 8, pp. 2795-2799.

DHAUNDIYAL, A. - ABDULRAHMAN, T. M. - LASZLO, T. 2019a. Thermo-kinetics of forest waste using model-free methods. In Universitas Scientiarum, vol. 24, no. 1, pp. 1-31.

DHAUNDIYAL, A. - SINGH, S. B. - HANON, M. M. 2018b. Study of distributed activation energy model using bivariate distribution function, $f(E 1, E 2)$. In Thermal Science and Engineering Progress. vol. 5, pp. 388-404.

DHAUNDIYAL, A. - TOTH, L. 2020. Modeling of hardwood pyrolysis using the convex combination of the mass conversion points. In Journal of Energy Resources Technology, Transactions of the ASME, vol. 142, no. 6, pp. 1-10.

DHAUNDIYAL, A. - SINGH, S. B. - HANON, M. M. - RAWAT, R. 2018c Determination of kinetic parameters for the thermal decomposition of Parthenium hysterophorus. In Environmental and Climate Technologies, vol. 22, no. 1, pp. 5-21.

DHAUNDIYAL, A. - SINGH, S. B. - ATSU, D. - DHAUNDIYAL, R. 2019b. Application of Monte Carlo simulation for energy modelling. In ACS Omega, vol. 4, no. 3, pp. 4984-4990.

DHAUNDIYAL, A. - TOTH, L. - BACSKAI, I. - ATSU, D. 2020. Analysis of pyrolysis reactor for hardwood (acacia) chips. In Renewable Energy, vol. 147, pp. 1979-1989.

DHAUNDIYAL, A. - SINGH, S. B. - HANON, M. M. - SHREMPF. N. 2018a. Clayton copula as an alternative perspective of multireaction model. In Environmental and Climate Technologies, vol. 22, no. 1, pp. 83-106.
DHAUNDIYAL, A. - SINGH, S. B. 2019. Stochastic analysis of multireaction model for non-linear thermal history. In Acta Technologica Agriculturae, vol. 22, no. 3, pp. 92-98.

FOGLER, H. S. 2004. Chemical reaction engineering. In the Engineering Handbook, second edition, pp. 79-1-79-18.

KUNG, H. C. 1972. A mathematical model of wood pyrolysis. In Combustion and Flame, vol. 18, no. 2, pp. 185-195.

LIM, S. M. - CHEW, M. Y. L. 2005. Compensation effects in the nonisothermal pyrolysis of wood. In Fire Safety Science, vol. 8, pp. 1109-1120.

MAA, P. S. - BAILIE, R. C. 1973. Influence of particle sizes and environmental conditions on high temperature pyrolysis of cellulosic material (theoretical). In Combustion Science and Technology, vol. 7, pp. 257-269.

MANI, T. - MURUGAN, P. - MAHINPEY, N. 2009. Determination of distributed activation energy model kinetic parameters using simulated annealing optimization method for nonisothermal pyrolysis of lignin. In Industrial and Engineering Chemistry Research, vol. 48, no. 3, pp. 1464-1467.

MÜLLER-HAGEDORN, M. - BOCKHORN, H. - KREBS, L. - MULLER, U. 2003. A comparative kinetic study on the pyrolysis of three different wood species. In Journal of Analytical and Applied Pyrolysis, pp. 231-249.

POLESEK-KARCZEWSKA, S. - KARDAŚ, D. 2015. Prediction of thermal behaviour of pyrolyzed wet biomass by means of model with inner wood structure. In Journal of Thermal Science, vol. 24, no. 1, pp. 82-89.

ROBERTS, A. F. - CLOUGH, G. 1963. Thermal decomposition of wood in an inert atmosphere. In Symposium (International) on Combustion, vol. 9, no. 1, pp. 158-166.

SADHUKHAN, A. K. - GUPTA, P. - SAHA, R. K. 2009. Modelling of pyrolysis of large wood particles. In Bioresource Technology, vol. 100, no. 12, pp. 3134-3139.

TINNEY, E. R. 2007. The combustion of wooden dowels in heated air. In Symposium (International) on Combustion, vol. 10, no.1, pp. 925-930.

WITKOWSKI, A. - STEC, A. - HULL, T. R. 2016. Thermal decomposition of polymeric materials. In SFPE Handbook of Fire Protection Engineering, fifth edition, pp. 167-254. 